\title{
Mucopolisacaridosis: generalidades y compromiso cardiovascular
}

\author{
Mucopolysaccharidosis: generalities and cardiovascular compromise \\ Claudia Stapper ${ }^{1,2}$ y Martha L. Solano $0^{1,2 *}$ \\ ${ }^{1}$ Servicio de Neuropediatría; ${ }^{2}$ Servicio de Pediatría y Cardiología Pediátrica. Fundación Cardioinfantil, Bogotá, Colombia
}

\section{Resumen}

Las mucopolisacaridosis (MPS) son un grupo de enfermedades de depósito lisosomal, monogénicas y de compromiso multisistémico, que en su mayoría se transmiten con un patrón de herencia autosómico recesivo, excepto la MPS II, que tiene un patrón ligado al cromosoma $X$. Sobre el sistema cardiovascular tienen un impacto variable, dependiendo del glucosaminoglicano que no se metaboliza y que, por ende, se acumula en el tejido valvular, miocárdico o vascular, generando una respuesta inflamatoria que produce fibrosis y deterioro estructural y funcional del tejido afectado. Así, en las variedades de MPS en que se depositan dermatán sulfato y condroitín sulfato existe un mayor compromiso del sistema cardiovascular. Dentro de este grupo están los pacientes con MPS I, II, IV, VI y VII, que inician la afección cardiaca en los primeros años de la vida con deterioro progresivo de su capacidad funcional y complicaciones tempranas, especialmente valvulopatía mitral o aórtica de grado variable, miocardiopatía infiltrativa y, a largo plazo, vasculopatía aórtica y coronaria, además del compromiso del sistema de conducción. Por lo tanto, a lo largo de su vida requieren seguimiento cardiológico y multidisciplinario periódico para detectar, tratar y evitar las múltiples complicaciones que puedan presentar y mejorar su pronóstico y calidad de vida. En la actualidad existe terapia de reemplazo enzimático, que iniciada de manera temprana mejora el fenotipo del paciente, su talla final y la capacidad funcional, y reduce el compromiso miocárdico. Lamentablemente, los tratamientos disponibles no poseen la misma efectividad para las manifestaciones valvulares, y por esta razón se continúa en la búsqueda de nuevos tratamientos que superen esta limitación.

Palabras clave: Mucopolisacaridosis. Corazón. Valvulopatía. Aortopatía.

\section{Abstract}

The mucopolysaccharidoses (MPS) are a group of monogenic lysosome storage disorders that produce multisystemic compromise. They are usually transmitted in autosomic recessive fashion, with MPS II as an exception, given its X-linked heredity pattern. At a cardiovascular level, these diseases have a variable impact that depends on the glycosaminoglycan that cannot be metabolized and that is therefore accumulated in the valvular, myocardial, or vascular tissues; this generates an inflammatory response that produces fibrosis, and structural and functional deterioration of the tissue. Hence, in the MPS

\section{Correspondencia:}

*Martha L. Solano

E-mail: marthasolano0@gmail.com
Disponible en internet: 28-06-2021 Rev Colomb Cardiol. 2021;28(Supl 2):3-11 www.rccardiologia.com 0120-5633 / C 2021 Sociedad Colombiana de Cardiología y Cirugía Cardiovascular. Publicado por Permanyer. Este es un artículo open access bajo la licencia CC BY-NC-ND (http://creativecommons.org/licenses/by-nc-nd/4.0/). 
types where dermatan sulfate and chondroitin sulfate are deposited, there is a higher compromise of the cardiovascular system. MPS I, II IV, VI, and VII are in this group; patients with these types of MPS initiate heart deterioration in the first years of their life with a progressive alteration of their functional capacity and early complications, especially with mitral or aortic valvular disease of variable grades, infiltrative myocardiopathy and, in the long term, aortic and coronary vasculopathy, in addition to compromise of the conduction system. Thus, these patients require a close monitoring of their cardiovascular system and periodic multisystemic following throughout their lifetime to detect, treat and prevent the multiple complications that could arise, and this way ameliorate their prognostic and life quality. Presently, there are enzyme replacement treatments that can treat the patient in certain areas - e. g.: enhancing their final height, helping their functional capacity and reducing their myocardial compromise. Unfortunately, these treatments offer no significant help at a valvular level and, for this reason, the search for new treatments that overcome this limitation is still ongoing.

Key words: Mucopolysaccharidosis. Cardiac disease. Valve disease. Aortopathy.

\section{Generalidades}

Las mucopolisacaridosis (MPS) constituyen un grupo de enfermedades causadas por la deficiencia de enzimas lisosomales específicas que participan en la vía catabólica de los glucosaminoglicanos (GAG) dermatán sulfato, heparán sulfato, queratán sulfato, condroitín sulfato y ácido hialurónico. Todas las MPS se transmiten con un patrón de herencia autosómico recesivo, excepto la MPS II, que se hereda ligada al cromosoma X (Tabla 1) 1 .

El acúmulo de GAG no degradados en los lisosomas provoca una disfunción de diferentes vías celulares que activan mecanismos de apoptosis y llevan a la muerte celular. A esto se añade la puesta en marcha de fenómenos inflamatorios que participan de forma significativa en el daño tisular progresivo².

\section{Manifestaciones clínicas}

Las MPS son enfermedades crónicas, multisistémicas y de carácter progresivo. Desde el punto de vista clínico se comportan de forma heterogénea y en cada tipo de MPS podemos evidenciar subtipos según la velocidad de progresión de la enfermedad, la edad de inicio de sus signos y síntomas, y la afectación o no del sistema nervioso central (SNC) de una forma primaria $^{3}$.

Acorde a lo anterior, podemos distinguir una presentación clásica con fenotipo típico o hurleriano, rasgos toscos, hipoacusia progresiva, disostosis ósea que lleva a deformidades como la mano en garra, talla baja y compromiso visceral importante que incluye afección cardiaca temprana con valvulopatía, infiltración miocárdica y alteración vascular, junto con un cuadro de obstrucción bronquial recurrente, que se presenta en los pacientes con las formas graves de MPS I y II, además de en los tipos VI y VII.
En segundo lugar está la presentación con alteraciones principalmente óseas, sin compromiso primario del SNC, pero con marcada disostosis, deformidad esquelética, patrón pulmonar mixto obstructivo y restrictivo por deformidad torácica, malformaciones vertebrales, displasia de caderas y genu valgo, y con compromiso cardiaco dado por infiltración miocárdica y cambios vasculares. En este grupo encontramos pacientes con enfermedad de Morquio A y $\mathrm{B}^{1}$.

El tercer subgrupo corresponde a pacientes con manifestaciones neurodegenerativas, con mayor compromiso del SNC y menor afectación sistémica, con un trastorno conductual importante. En este grupo encontramos los pacientes con síndrome de Sanfilippo A, B, $C$ y D. La mayoría de los pacientes tienen un fenotipo normal al nacimiento y los signos y síntomas se van evidenciando en los primeros meses de vida para las formas de presentación rápidamente involutivas o graves, y en la infancia tardía o la adolescencia para las formas atenuadas ${ }^{4}$.

\section{Diagnóstico}

Aunque la mayoría de los pacientes con MPS tienen rasgos fenotípicos característicos, estos aparecen evolutivamente, lo que en muchas ocasiones retrasa el diagnóstico. Además, existen pacientes con formas atenuadas, con afectación tardía o limitada a algunos sistemas $^{5}$. En la esfera cardiovascular podremos encontrar hallazgos casi aislados o predominantes de valvulopatía (lo más frecuente), hipertrofia miocárdica o tardíamente dilatada, afectación coronaria, aortopatía o hipertensión pulmonar (por depósito de GAG en los vasos pulmonares o por afectación pulmonar).

Además, el patrón de herencia (ligada al cromosoma X para el tipo II y autosómica recesiva para el resto de los tipos) genera árboles genealógicos relativamente silentes. 
Tabla 1. Clasificación de las mucopolisacaridosis

\begin{tabular}{|l|l|l|}
\hline Tipo & Enzima deficiente & Nombre \\
\hline I & L-iduronidasa & Hurler-Scheie \\
\hline II & Iduronato 2 sulfatasa & $\begin{array}{l}\text { Hunter con y sin } \\
\text { compromiso de sistema } \\
\text { nervioso central }\end{array}$ \\
\hline III & $\begin{array}{l}\text { Heparán N sulfatasa } \\
\text { N-acetilglucosaminidasa } \\
\text { Glucosamida } \\
\text { acetiltransferasa } \\
\text { N-acetilglucosamina-6- } \\
\text { sulfatasa }\end{array}$ & $\begin{array}{l}\text { Sanfilippo A } \\
\text { Sanfilippo B }\end{array}$ \\
\hline IV A & $\begin{array}{l}\text { Galactosa 6 sulfatasa } \\
\text { Sanfilippo D }\end{array}$ \\
\hline IV B & B galactosidasa & Morquio A \\
\hline VI & Arilsulfatasa B & Morquio B \\
\hline VII & B glucuronidasa & Maroteaux-Lamy \\
\hline
\end{tabular}

Dado que la eficacia del tratamiento enzimático sustitutivo es más alta si este se instaura de modo precoz, es importante elevar el índice de sospecha.

Rigoldi et al. ${ }^{5}$ proponen algunos signos de alarma (red flags) para formas tardías o atenuadas (Tabla 2).

En los pacientes con sospecha clínica se debe realizar la determinación cualitativa y cuantitativa de GAG en orina, preferiblemente en orina de 24 horas; si el patrón encontrado es sugestivo de MPS, el paso siguiente es corroborar el diagnóstico y definir el tipo específico identificando la enzima deficiente en leucocitos, plasma o fibroblastos ${ }^{4}$. Cabe anotar que, aun cuando la confirmación diagnóstica se hace con el estudio enzimático, los GAG urinarios son marcadores biológicos que nos dan información no solo del curso natural de la enfermedad, sino también de la posible respuesta al tratamiento ${ }^{4}$.

\section{Tratamiento}

Teniendo en cuenta el compromiso sistémico y progresivo de las MPS, el abordaje de estos pacientes requiere un equipo multidisciplinario que debe establecer objetivos terapéuticos en cada caso. El equipo debe incluir especialistas en pediatría, cardiología, neuropediatría, neumología, fisiatría (rehabilitación), genética, psicología y otras especialidades quirúrgicas tales como anestesia (con entrenamiento en vía aérea difícil), neurocirugía, ortopedia, cirugía cardiovascular, oftalmología y otorrinolaringología.

Acorde con la literatura mundial, las principales causas de fallecimiento de estos pacientes son las complicaciones infecciosas, la falla respiratoria, la
Tabla 2. Signos de alarma de mucopolisacaridosis

$$
\begin{aligned}
& \text { - Rigidez articular no inflamatoria } \\
& \text { - Síndrome del túnel carpiano } \\
& \text { - Valvulopatía } \\
& \text { - Opacificación corneal } \\
& \text { - Anomalías esqueléticas } \\
& \text { - Hernias } \\
& \text { - Hepatomegalia }
\end{aligned}
$$

enfermedad cardiaca y las complicaciones anestésicas $^{6}$. Los pacientes con MPS requieren a lo largo de su vida múltiples intervenciones quirúrgicas, por lo que es importante contar con un equipo de anestesia entrenado en vía aérea difícil para cualquier abordaje quirúrgico y los múltiples procedimientos diagnósticos que será necesario realizar?.

Con respecto al tratamiento farmacológico específi$\mathrm{co}$, los pacientes con MPS requieren terapia de reemplazo enzimático (TRE), que si bien a corto y largo plazo ha demostrado una estabilización o una mejoría en las cifras de GAG, la capacidad física del paciente y su función pulmonar, tiene como limitante que no atraviesa la barrera hematoencefálica, lo que limita su eficacia en los subtipos con compromiso primario del $\mathrm{SNC}^{8,9}$. Adicionalmente, la TRE, aunque ha logrado una mejoría en la infiltración miocárdica, no penetra al tejido valvular cardiaco, por lo que el compromiso valvular es progresivo y una proporción de pacientes requerirán tratamiento quirúrgico9 ${ }^{9}$

\section{Tratamientos en desarrollo}

La terapia génica para estos pacientes ha mostrado resultados parciales prometedores, pero aún se encuentra en fase de ensayos clínicos para demostrar su efectividad y seguridad. De otro lado, a la fecha hay varios ensayos clínicos de TRE con la capacidad de atravesar la barrera hematoencefálica para aquellos pacientes con compromiso primario del SNC. En la actualidad, el compromiso primario del SNC, del tejido óseo y del sistema cardiovascular constituye el reto a superar en el tratamiento de estas enfermedades ${ }^{10}$.

\section{Compromiso cardiovascular en las mucopolisacaridosis}

Una vez diagnosticada una MPS a un paciente es recomendable realizar una evaluación cardiológica integral para determinar si existe compromiso cardiovascular y planear el seguimiento que requerirá. Las MPS 
tienen un impacto variable en el sistema cardiovascular, dependiendo del GAG que no se metaboliza y que, por ende, se acumula en el tejido valvular, miocárdico, de conducción o vascular, generando una respuesta inflamatoria que lleva a fibrosis y deterioro estructural $\mathrm{y}$ funcional del tejido afectado.

Así, en las variedades de MPS en que se depositan dermatán sulfato y condroitín sulfato, como las de tipo I (Hurler), tipo II (Hunter), tipo VI (Maroteaux-Lamy) y tipo VII (Sly), existe un compromiso temprano y progresivo del sistema cardiovascular, y es indispensable el acompañamiento cardiológico desde los primeros años y a lo largo de la vida del paciente. En la MPS de tipo IV (Morquio), el GAG no metabolizado es el queratán sulfato, lo que induce alteraciones articulares y esqueléticas dominantes, mientras que las alteraciones valvulares y vasculares suelen ser lentamente progresivas, y en estos pacientes también es necesario realizar seguimiento cardiológico, aunque a intervalos más largos ${ }^{11}$ (Tabla 3).

Es necesario conocer las alteraciones histopatológicas que induce el acúmulo de GAG en los diferentes tejidos del sistema cardiovascular para entender las implicaciones de su acúmulo sobre la estructura y la función de cada uno de ellos. A continuación resumiremos su patogénesis.

\section{Valvulopatía}

Constituye la alteración más frecuente (60-90\%) en los pacientes con MPS de los tipos I, II, VI y VII, y es la alteración menos modificable con la TRE. Las válvulas mitral y aórtica son las más comprometidas, presentando disrupción y desorganización del colágeno valvular, inflamación y fibrosis progresiva que lleva a un engrosamiento lineal o nodular de las valvas y del aparato subvalvular, un acortamiento de las cuerdas tendinosas y la infiltración de los músculos papilares con limitación progresiva de la motilidad valvular, ocasionando tempranamente insuficiencia valvular y a largo plazo estenosis ${ }^{12}$.

\section{Miocardiopatía}

Puede resultar de la asociación de tres factores principales: infiltración crónica de GAG en el endocardio y el miocardio que induce fibrosis lentamente progresiva, dando un aspecto de pseudohipertrofia y produciendo alteraciones en la relajación ventricular; en segundo lugar, la sobrecarga de volumen asociada a disfunción valvular mitral o aórtica; y en tercer lugar, la isquemia generada por el compromiso de las arterias coronarias.
Tabla 3. Mucopolisacaridosis y compromiso cardiaco

\begin{tabular}{|l|l|l|l|}
\hline Tipo & Valvulopatía & Miocardiopatía & $\begin{array}{l}\text { Alteración } \\
\text { vascular }\end{array}$ \\
\hline I & $\begin{array}{l}\text { Mitral y aórtica } \\
>80 \%\end{array}$ & $\begin{array}{l}\text { Infiltrativa } \\
65 \%\end{array}$ & $\begin{array}{l}\text { Aortopatía } 30 \% \\
\text { Coronariopatía }\end{array}$ \\
\hline II & $\begin{array}{l}\text { Mitral y aórtica } \\
65-75 \%\end{array}$ & $\begin{array}{l}\text { Infiltrativa } \\
48-55 \%\end{array}$ & $\begin{array}{l}\text { Aortopatía } 15 \% \\
\text { Coronariopatía }\end{array}$ \\
\hline III & No & No & No \\
\hline IV A & $\begin{array}{l}\text { Mitral y aórtica } \\
30-50 \%\end{array}$ & $\begin{array}{l}\text { Infiltrativa } \\
50-60 \%\end{array}$ & $\begin{array}{l}\text { Dilatación raíz } \\
\text { aórtica } 20 \%\end{array}$ \\
\hline VI & $\begin{array}{l}\text { Mitral y aórtica } \\
>95 \%\end{array}$ & $\begin{array}{l}\text { Infiltrativa } \\
>60 \%\end{array}$ & $\begin{array}{l}\text { Dilatación raíz } \\
\text { aórtica } \\
\text { Coronariopatía }\end{array}$ \\
\hline VII & $\begin{array}{l}\text { Mitral y aórtica } \\
50 \%\end{array}$ & $\begin{array}{l}\text { Infiltrativa } \\
\text { No descrita }\end{array}$ \\
\hline
\end{tabular}

\section{Vasculopatía}

Se ha descrito compromiso de las arterias coronarias en la mayoría de las MPS, pero es más frecuente, temprano y progresivo en la de tipo I y en segundo lugar en la de tipo II. En la porción epicárdica de estos vasos, el depósito de GAG en la capa íntima induce una proliferación difusa con obliteración progresiva de la luz del vaso, provocando isquemia miocárdica. Algunos estudios sugieren, además, el desarrollo temprano de aterosclero$\mathrm{sis}^{13}$. En los pacientes con MPS de los tipos II y VI se ha descrito también una oclusión progresiva de las arteriolas coronarias, especialmente en la región apical del ventrículo izquierdo, que lleva a la formación de aneurismas. Los grandes vasos pueden presentar engrosamiento de sus paredes, pérdida de la elasticidad y oclusión lentamente progresiva. En los pacientes con MPS de los tipos I y II se ha encontrado, hasta en un $30 \%$ de los casos, estrechamientos de la aorta torácica y abdominal, sobre todo en la región ístmica, que producen un cuadro clínico similar al de una coartación aórtica y que generalmente requieren tratamiento correctivo. En las MPS de tipos I, IV y VII puede producirse dilatación de la raíz aórtica y de la aorta ascendente relacionada con el incremento en la degradación de la elastina. También se ha encontrado, en pacientes con MPS de tipo IV, una tortuosidad importante de la aorta descendente abdominal y las arterias carótidas, subclavias, vertebrales y cerebrales ${ }^{14}$.

\section{Compromiso del sistema de conducción}

Se presenta principalmente en las MPS de tipos I y II, pues el acúmulo de GAG en las células de los nodos 
sinusal y atrioventricular, así como en las que componen el haz de Hiss y sus ramas, produce fibrosis y hialinización lentamente progresiva, lo que lleva a una alteración de la conducción eléctrica intracardiaca.

\section{Manifestaciones clínicas de compromiso cardiovascular}

Lo ideal sería retardar al máximo la aparición de síntomas de compromiso cardiovascular mediante la realización de un diagnóstico en el primer año de vida $y$ el inicio de TRE, pero lamentablemente en nuestro medio el diagnóstico de las MPS suele ser tardío por su baja frecuencia de presentación, la falta de reconocimiento por parte del personal médico y las barreras generadas por el sistema de salud, así que, en general, cuando evaluamos al paciente ya existen signos y síntomas de alteración cardiovascular.

La disnea suele ser la primera manifestación clínica en los pacientes con MPS de los tipos I II, IV, VI y VII. Es de etiología mixta, pues además de las alteraciones cardiacas existe compromiso importante de la vía aérea superior e inferior, y deformidad variable de la caja torácica, lo cual reduce la capacidad funcional pulmonar. Con frecuencia se presentan palpitaciones, asociadas a taquicardia sinusal secundaria a desacondicionamiento físico crónico inherente a la poca actividad física que la mayoría de los pacientes puede realizar, o como manifestación de sobrecarga auricular o ventricular por valvulopatía. El dolor torácico de tipo anginoso suele aparecer en los pacientes no tratados a partir de la segunda década de la vida, y es un indicador de compromiso miocárdico o coronario importante. De igual manera, la presencia de síncope es una señal de alto riesgo y sugiere compromiso del sistema de conducción que puede llevar a la muerte súbita.

En la exploración física son evidentes la facies tosca, la talla baja patológica y diferentes alteraciones articulares y esqueléticas, como se mencionó previamente. Es frecuente encontrar taquicardia sinusal, taquipnea y oximetría normal en la fase inicial. La presencia de hipertensión arterial en los pacientes con MPS en general es del $2 \%$, pero en la de tipo II puede presentarse con una frecuencia tan alta como el $25 \%$ en los primeros 18 años de vida ${ }^{15}$.

El tórax muestra grados variables de deformidad, con incremento en el diámetro anteroposterior, pectus carinatum en algunos casos, horizontalización de las costillas y reducción de los espacios intercostales; el punto de máximo impulso, si no hay compromiso valvular importante, suele estar normalmente localizado, pero si existe dilatación ventricular izquierda muestra un desplazamiento inferolateral. En la auscultación es frecuente encontrar un soplo de insuficiencia mitral $o$ insuficiencia aórtica (o ambos), y el segundo ruido cardiaco de intensidad normal si no hay hipertensión pulmonar o aumentado en caso de que se presente.

En la exploración abdominal se puede encontrar hepatomegalia congestiva en los pacientes con MPS de los tipos I, II, VI y VII no tratados y con disfunción biventricular importante. Es fundamental, en todos los casos, realizar una palpación comparativa de los pulsos braquiales, radiales, femorales, pedios y tibiales posteriores, y determinar si existen asimetrías. Ante el incremento en la amplitud de los correspondientes a los miembros superiores y la disminución de los inferiores, se recomienda realizar la medición de la presión arterial en las cuatro extremidades para descartar la presencia de una coartación aórtica secundaria a compromiso vascular por la MPS.

\section{Métodos diagnósticos}

En la evaluación cardiológica de los pacientes con MPS se deben realizar estudios para objetivar el impacto de la enfermedad sobre la estructura y la función cardiacas, evaluar la respuesta a la TRE y definir la necesidad de tratamiento farmacológico o quirúrgico.

\section{Radiografía de tórax}

Es una herramienta útil en el seguimiento de los pacientes para valorar la configuración del tórax, el parénquima y la vascularización pulmonar, el tamaño y la configuración de la silueta cardiaca, que se van alterando progresivamente según el grado de afectación valvular, miocárdica, vascular o pulmonar (Fig. 1).

\section{Electrocardiograma}

En las fases iniciales muestra taquicardia sinusal con intervalos y voltajes normales para la edad, y con frecuencia existe un bloqueo incompleto de rama derecha. A medida que progresa la enfermedad, y especialmente si el paciente no recibe tratamiento con TRE, se presentan signos de sobrecarga auricular y ventricular izquierda secundaria a la valvulopatía mitral y aórtica (Fig. 2), y en fases tardías se observan una reducción progresiva en el voltaje del QRS, una prolongación de los intervalos PR y del QT, y finalmente 


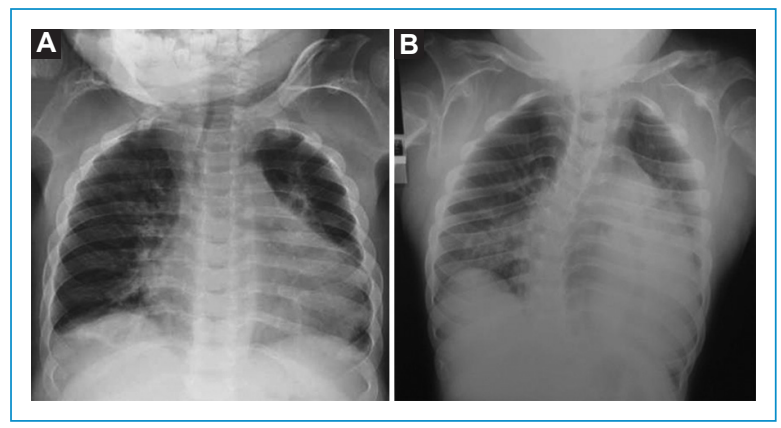

Figura 1. Radiografía de tórax de un paciente con mucopolisacaridosis de tipo II. A: a los 10 años de edad, cardiomegalia moderada. B: a los 16 años, cardiomegalia importante y congestión venosa pulmonar. (Imágenes de archivo personal).

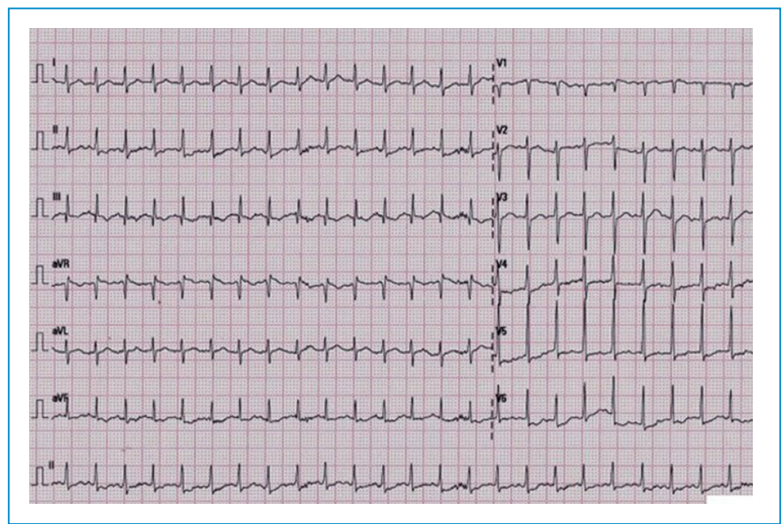

Figura 2. Electrocardiograma de un paciente con mucopolisacaridosis de tipo VI con taquicardia sinusal y signos de sobrecarga auricular y ventricular izquierda por disfunción valvular mitral importante. (Imagen de archivo personal).

un bloqueo atrioventricular de segundo y de tercer $\operatorname{grado}^{16}$.

\section{Ecocardiograma transtorácico}

Es una prueba no invasiva que permite el diagnóstico $y$ el seguimiento de las alteraciones valvulares y del desarrollo de hipertrofia, dilatación y disfunción de cavidades. Sus principales limitaciones en los pacientes con MPS son la frecuente falta de colaboración en caso de afectación neurológica, así como la limitación frecuente de la ventana acústica en relación con la configuración de la caja torácica y las alteraciones pulmonares. La ecocardiografía transesofágica es poco utilizada en estos pacientes por los riesgos respiratorios derivados de la sedación y la intubación.

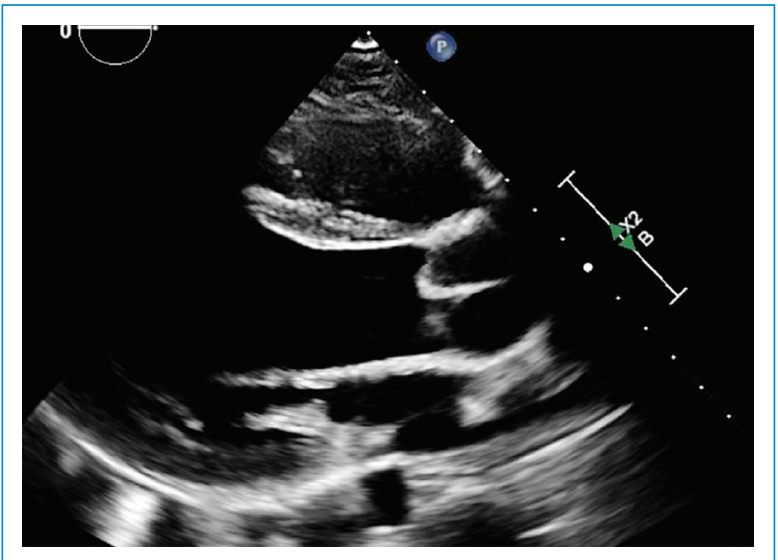

Figura 3. Vista paraesternal en el eje largo que muestra un engrosamiento de las valvas aórticas y mitrales, especialmente de la valva posterior, en un paciente con mucopolisacaridosis de tipo II. (Imagen de archivo personal).

La tecnología de speckle-tracking aplicada a la medición del strain longitudinal global podría tener utilidad en estos pacientes. Lin et al. ${ }^{17}$ muestran una mayor precocidad en el diagnóstico de disfunción ventricular en los pacientes con MPS que con los parámetros ecocardiográficos convencionales.

En los primeros años de vida, los pacientes con MPS de los tipos I, II, VI y VII muestran un engrosamiento leve de las valvas mitrales y de su aparato subvalvular y de las valvas aórticas, con función normal o levemente alterada (Fig. 3). Conforme pasa el tiempo, dado que la TRE no penetra al tejido valvular, las alteraciones empeoran de manera progresiva y llevan a un engrosamiento moderado e importante, en especial, de las zonas de coaptación valvular y de las cuerdas tendinosas, las cuales terminan acortándose y limitando gravemente la motilidad valvular, lo que genera insuficiencia mitral y aórtica de gravedad creciente que a su vez induce dilatación de la aurícula y del ventrículo izquierdo. De manera concomitante, se producen un aumento de la refringencia miocárdica y un aumento del espesor de la pared ventricular, conduciendo a disfunción diastólica temprana y sistólica tardía ${ }^{18,19}$.

Por lo anterior, se recomienda el uso de strain para la detección y el manejo precoz de la disfunción ventricular, aunque en algunos pacientes resulta difícil por la inadecuada ventana ecocardiográfica ${ }^{17}$.

En los pacientes con MPS de los tipos I, IV y VII, después de la segunda década de la vida se observa dilatación del anillo, de la raíz aórtica y de la unión sinotubular junto con la aorta ascendente; se sugiere determinar en cada evaluación el valor $\mathbf{Z}$ de dichas 
estructuras para definir la gravedad de la dilatación. Igualmente, se debe analizar con Doppler continuo el flujo en la aorta descendente a nivel del istmo y distalmente, puesto que existe el riesgo de coartación adquirida a largo plazo.

\section{Test de la marcha de 6 minutos}

Es una prueba útil para la determinación de la clase funcional de los pacientes con MPS, quienes por sus alteraciones osteomusculares no pueden realizar una prueba de esfuerzo convencional. Facilita el seguimiento de la función musculoesquelética, cardiovascular y pulmonar, permite evaluar la respuesta a la TRE y es un indicador de calidad de vida y de supervivencia, por lo que se recomienda realizarla anualmente.

\section{Holter de 24 horas}

Está indicado en los pacientes con palpitaciones recurrentes o dolor torácico anginoso, pues permite detectar la presencia de arritmias, trastornos de la conducción y trastornos de la repolarización sugestivos de isquemia. Igualmente se recomienda en los pacientes con disfunción valvular mitral o aórtica con dilatación importante de cavidades, en los que se favorece la aparición de arritmias o disfunción ventricular, para considerar la necesidad de tratamiento quirúrgico.

\section{Monitoría ambulatoria de la presión arterial}

Debe realizarse en los pacientes que presenten hipertensión arterial, alteraciones en los pulsos periféricos 0 un gradiente medio en la aorta descendente $>20 \mathrm{mmHg}$.

\section{Resonancia magnética y tomografía computarizada}

Son estudios complementarios recomendados en los pacientes con MPS de los tipos I, IV y VII con signos clínicos o ecocardiográficos de compromiso aórtico y de los grandes vasos, para determinar la necesidad de manejo intervencionista o quirúrgico (Fig. 4).

La resonancia magnética aporta información sobre los volúmenes y la función de las cavidades en pacientes en quienes la ecocardiografía no es concluyente, y ayuda a cuantificar la fracción regurgitante en las valvulopatías. También puede tener interés en la caracterización tisular en los casos de infiltración miocárdica.

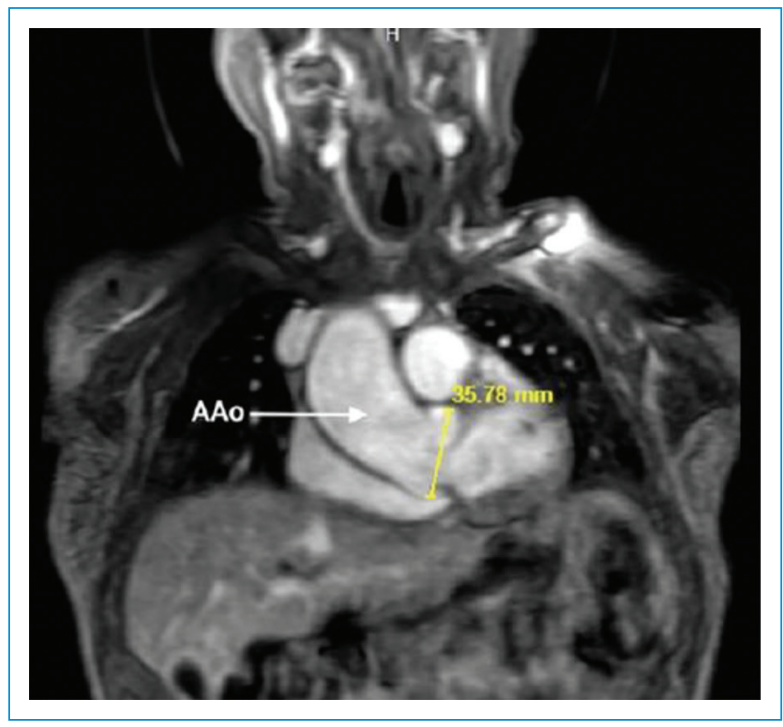

Figura 4. Resonancia magnética que muestra una dilatación de la raíz aórtica y de la AAo. (Imagen de archivo personal). AAo: aorta ascendente.

Entre sus limitaciones hay que señalar la frecuencia de taquicardia en estos pacientes, lo que limita su resolución, así como la necesidad de sedación en los pacientes con mala colaboración.

La tomografía computarizada permite, con tiempos de adquisición mucho más cortos, realizar medidas precisas en las alteraciones de la aorta y de los grandes vasos, y evaluar alteraciones coronarias, frecuentes en estos pacientes, aunque el patrón de afectación es diferente al de la enfermedad aterosclerótica, por lo que tanto la tomografía computarizada coronaria como la coronariografía pueden pasar por alto algunas alteraciones en estos pacientes ${ }^{11}$.

\section{Cateterismo cardiaco}

Está indicado en los pacientes con signos de isquemia miocárdica y sospecha de compromiso coronario, así como en los candidatos a reemplazo valvular aórtico o mitral. También se ha utilizado para la realización de angioplastia aórtica y colocación de stent en los pacientes con coartación adquirida.

\section{Tratamiento médico}

Además de la TRE y las otras opciones de tratamiento ya mencionadas, los pacientes con MPS que presenten valvulopatía mitral o aórtica (o ambas), con insuficiencia o estenosis (o ambas), deben recibir 
profilaxis frente a la endocarditis infecciosa en los procedimientos que puedan generar bacteriemia, y si el grado de disfunción valvular tiene repercusión hemodinámica se debe iniciar tratamiento con diuréticos (furosemida o espironolactona) e inhibidores de la enzima convertidora de la angiotensina (captopril o enalapril) a dosis habituales. El uso de betabloqueadores debe ser cuidadoso, en especial en los pacientes con MPS de tipo IV, que con frecuencia no toleran reducciones en su frecuencia cardiaca. Se están realizando estudios con antagonistas del receptor de la angiotensina II, como el losartán, en pacientes con MPS y aortopatía, con resultados favorables, pero aún sin la suficiente evidencia ${ }^{20,21}$.

\section{Manejo quirúrgico}

En los pacientes que presenten insuficiencia mitral de grado IV o estenosis con un gradiente medio $>10 \mathrm{mmHg}$, arritmias auriculares o ventriculares secundarias, e hipertensión pulmonar, se debe realizar un remplazo valvular con prótesis mecánicas. Lo mismo aplica para los pacientes con insuficiencia valvular aórtica de grado IV 0 estenosis con un gradiente medio $>40 \mathrm{mmHg}$. Estos procedimientos deben ser realizados en centros de excelencia en la atención cardiovascular, teniendo en cuenta la gran comorbilidad de los pacientes con MPS, especialmente la vía aérea difícil.

La afectación es mucho más frecuente en la válvulas izquierdas, sobre todo en la mitral, y es más habitual la insuficiencia que la estenosis. La afectación no se limita a los velos, pues también presenta compromiso el aparato subvalvular y hay calcificación, por lo que a menudo no es posible la reparación, precisando sustitución con prótesis mecánica valvular o aórtica (o ambas $)^{6}$. Sin embargo, es importante individualizar algunos factores, como los riesgos de la anticoagulación en pacientes con afectación neurológica más proclives a traumatismos, y la esperanza de vida del paciente. Es importante recalcar la prácticamente nula eficacia de TRE en la afectación valvular.

La sustitución aórtica mediante cirugía de Ross, frecuente en niños y jóvenes con otras patologías, está contraindicada en los pacientes con MPS por la afectación de la raíz pulmonar nativa ${ }^{11}$.

\section{Seguimiento}

Todos los pacientes con MPS de los tipos I, II, IV, VI y VII deben ser evaluados clínicamente por cardiología con electrocardiograma y ecocardiograma transtorácico, por lo menos una vez al año. A medida que aparecen las alteraciones cardiovasculares, y según la sintomatología del paciente, se requerirán los estudios complementarios antes mencionados. Cuando la disfunción valvular mitral o aórtica sea moderada o importante será necesario realizar evaluaciones a intervalos de 3-6 meses, para evaluar un posible ajuste medicamentoso y definir la necesidad de manejo quirúrgico ${ }^{22}$.

\section{Conclusiones}

Las MPS son las enfermedades de depósito lisosomal más frecuentes, son monogénicas, producen un compromiso multisistémico progresivo y se transmiten con un patrón de herencia autosómico recesivo, excepto la de tipo II, que está ligada al cromosoma X. En cuanto a sus manifestaciones cardiovasculares, las MPS afectan preferencialmente el tejido valvular mitral y aórtico, el miocardio, la aorta y sus principales ramas, y las arterias coronarias, y con menor frecuencia el tejido de conducción. Los tipos I, II y VI producen valvulopatía y miocardiopatía tempranas y de mayor gravedad; el tipo IV tiene un comportamiento intracardiaco menos agresivo, pero con frecuencia afecta la aorta y los vasos del cuello. Con un diagnóstico temprano, la TRE iniciada antes de los 4 años mejora el fenotipo y la talla final, mantiene la clase funcional y retarda 0 reduce el compromiso infiltrativo miocárdico, pero es poco efectiva en la afectación valvular. Por tal motivo, los pacientes con MPS deben tener un seguimiento cardiológico integral a lo largo de su vida, recibir el tratamiento farmacológico o quirúrgico que requieran sus lesiones, y ser atendidos por un equipo multidisciplinario que favorezca una mejor calidad de vida.

\section{Conflicto de intereses}

Las autores declaran que no existe conflicto de intereses.

\section{Responsabilidades éticas}

Protección de personas y animales. Los autores declaran que para esta investigación no se han realizado experimentos en seres humanos ni en animales.

Confidencialidad de los datos. Los autores declaran que han seguido los protocolos de su centro de trabajo sobre la publicación de datos de pacientes. 
Derecho a la privacidad y consentimiento informado. Los autores declaran que en este artículo no aparecen datos de pacientes.

\section{Bibliografía}

1. Giugliani R, Federhen A, Muñoz M, Vieira T, Artigalás $O$, Lapagesse L. Mucopolysaccharidosis I, II, and VI: brief review and guidelines for treatment. Genet Mol Biol. 2010;33:589-604

2. Tomatsu S, Montaño AM, Oikawa H, Smith M, Barrera L, Chinen $\mathrm{Y}$, et al. Mucopolysaccharidosis type IVA (Morquio A disease): clinical review and current treatment. Curr Pharm Biotechnol. 2011;12:931-45.

3. Hendriksz CJ, Al-Jawad M, Berger KI, Hawley SM, Lawrence R, Mc Ardle $\mathrm{C}$, et al. Clinical overview and treatment options for non-skeletal manifestations of mucopolysaccharidosis type IVA. J Inherit Metab Dis. 2013;36:309-22.

4. Hendriksz CJ, Giugliani R, Harmatz P, Lampe C, Martins AM, Pastores $\mathrm{GM}$, et al. Design, baseline characteristics, and early findings of the MPS VI (mucopolysaccharidosis VI) Clinical Surveillance Program (CSP) J Inherit Metab Dis. 2013;36:373-84

5. Rigoldi M, Verrecchia E, Manna R, Mascia MT. Clinical hints to diagnosis of attenuated forms of mucopolysaccharidoses. Ital J Pediatr. 2018;44(Suppl 2):132.

6. Solanki GA, Alden TD, Burton BK, Giugliani R, Horovitz DD, Jones SA, et al. A multinational, multidisciplinary consensus for the diagnosis and management of spinal cord compression among patients with mucopolysaccharidosis VI. Mol Genet Metab. 2012;107:15-24.

7. Harmatz P, Whitley CB, Waber L, Pais R, Steiner R, Plecko B, et al. Enzyme replacement therapy in mucopolysaccharidosis VI (Maroteaux-Lamy syndrome). J Pediatr. 2004:144:574-80.

8. Harmatz P, Yu ZF, Giugliani R, Schwartz IV, Guffon N, Teles EL, et al. Enzyme replacement therapy for mucopolysaccharidosis VI: evaluation of long-term pulmonary function in patients treated with recombinant human $\mathrm{N}$-acetylgalactosamine 4-sulfatase. J Inherit Metab Dis. 2010;33:51-60.

9. Muenzer J, Wraith JE, Beck M, Giugliani R, Harmatz P, Eng CM, et al. A phase II/III clinical study of enzyme replacement therapy with idursulfase in mucopolysaccharidosis II (Hunter syndrome). Genet Med. 2006;8:465-73.
10. Noh H, Lee Jl. Current and potential therapeutic strategies for mucopolysaccharidoses. J Clin Pharm Ther. 2014;39:215-24.

11. Braunlin E, Harmatz $P$, Scarpa M, Furlanetto B, Kampmann C, Loehr J, et al. Cardiac disease in patients with mucopolysaccharidosis: presentation, diagnosis and management. J Inherit Metab Dis. 2011; 34:1183-97.

12. Kampmann C, Beck M, Morin I, Loehr J. Prevalence and characterization of cardiac involvement in Hunter syndrome. J Pediatr. 2011;159:327-331.e2.

13. Canda E, Köse M, Kağnıcı M, Dondurmac M, Kalkan Uçar S, Sözmen E, et al. Evaluation of cardiovascular involvement and cytokine levels in patients with mucopolysaccharidosis. J Pedatr Res. 2019;6:121-7.

14. Powell A, Taylor M, Burrow T, Hopkin R, Prada C, Jefferies J. Widespread vasculopathy in a patient with Morquio A syndrome. Tex Heart Inst J. 2017:44:420-3.

15. Suárez-Guerrero J, Gómez Higuera P, Arias Flórez J, Contreras-García G. Mucopolisacaridosis: características clínicas, diagnóstico y de manejo. Rev Chil Pediatr. 2016;87:295-304.

16. Chow LTC, Chow WH. The cardiac conduction system in Hurler syndrome: pathological features and clinical implications. Cardiol Young. 1992:2:196-9.

17. Lin HY, Chuang CK, Lee CL, Chen MR, Sung KT, Lin SM, et al. Cardiac evaluation using two-dimensional speckle-tracking echocardiography and conventional echocardiography in Taiwanese patients with mucopolysaccharidoses. Diagnostics (Basel). 2020;10:62.

18. Andrade M, Guimarães I, Acosta A, Leão E, Moreira M, Mendes C. Left ventricular assessment in patients with mucopolysaccharidosis using conventional echocardiography and myocardial deformation by two-dimensional speckle-tracking method. J Pediatr. 2019;95:475-81.

19. Fesslová V, Corti P, Sersale G, Rovelli A, Russo P, Mannarino S, et al. The natural course and the impact of therapies of cardiac involvement in the mucopolysaccharidoses. Cardiol Young. 2009;19:170-8.

20. Boffi L, Russo P, Limongelli G. Early diagnosis and management of cardiac manifestations in mucopolysaccharidoses: a practical guide for paediatric and adult cardiologists. Ital J Pediatr. 2018:44(Suppl 2):122.

21. Giugliani R, Villarreal M, Valdez C, Hawilou A, Guelbert N, Garzón L, et al. Guidelines for diagnosis and treatment of Hunter syndrome for clinicians in Latin America. Genet Mol Biol. 2014;37:315-29.

22. Encarnacion CO, Hang D, Earing M, Mitchell ME. Mucopolysaccharidoses causing valvular heart disease: report and review of surgical management. World J Pediatr Congenit Heart Surg. 2020;11:NP22-4. 